\section{Conservative Approach of Spontaneous Spinal Epidural Haematomas in the Era of Anticoagulant Treatments}

Authors: Ivana Stetkarova, ${ }^{1}$ Edvard Ehler, ${ }^{2}$ Karel Brabec, ${ }^{1}$ Lenka Jelinkova, ${ }^{1}$ Jiri Weichet, ${ }^{3}$ Leos Ungermann, ${ }^{4}$ Tomas Peisker ${ }^{1}$

1. Department of Neurology, Charles University and Faculty Hospital Kralovske Vinohrady, Prague, Czech Republic

2. Neurological Department, Pardubice University and Pardubice Regional Hospital, Pardubice, Czech Republic

3. Department of Radiology, Charles University and Faculty Hospital Kralovske Vinohrady, Prague, Czech Republic

4. Department of Radiology, Pardubice University and Pardubice Regional Hospital, Pardubice,

Czech Republic

*Correspondence to ivana.stetkarova@lf3.cuni.cz

Disclosure: The authors have declared no conflicts of interest.

Keywords: Anticoagulant therapy, spinal cord compression, spontaneous spinal epidural hematoma (SSEH), surgery.

Citation: EMJ Neurol. 2020;DOI/10.33590/

emjneurol/200914

\section{BACKGROUNDS AND AIMS}

Anticoagulation and antiplatelet therapy is a widespread management option in various indications, especially in elderly patients who are at greater risk of complications related to any treatment. One of the important and rare complications of anticoagulation and antiplatelet therapy is spontaneous spinal epidural haematoma (SSEH)., ${ }^{1,2}$ Surgery remains the gold standard treatment, especially in cases of progressive neurological deficit. ${ }^{3}$ In this study, a group of 14 patients with SSEH is presented. Additionally, the clinical symptoms of SSEH, diagnostic procedures, conservative and surgical treatments, and different risk factors are discussed.

\section{METHODS}

Between 2010 and 2019, 14 patients (age range: 17-89 years; 10 females) were hospitalised with SSEH in two large hospitals in the Czech Republic. Table 1 presents their demographic and clinical data, including MRI showing haematoma localisation, treatment efficacy, and risk factors with a focus on anticoagulant use. In addition to a detailed clinical neurological examination, the severity of spinal cord disability was assessed using the American Spinal Injury Association (ASIA) Impairment Scale (AIS) classification.

\section{RESULTS}

Eight patients received oral anticoagulant therapy (six warfarin, one dabigatran, one apixaban). There was an increase of international normalised ratio values above 3.0 in three patients using warfarin (two cases for atrial fibrillation, one for deep vein thrombosis). Eleven patients experienced arterial hypertension. Two patients were using acetylsalicylic acid of $100 \mathrm{mg} /$ day. In summary, 10 patients out of 14 (71\%) were given oral anticoagulant/antiplatelet therapy.

All patients reported acute onset of severe pain as the initial symptom, mostly in the lower cervical spine. Twelve patients reported some degree of neurological deficit accompanying the pain. Only two patients did not develop any neurological deficit. All patients underwent urgent MRI for the detection of SSEH. With respect to the affected level of the spine, the lower cervical/upper thoracic region was the most common site of involvement $(n=7 ; 50 \%)$. Six patients (43\%) underwent surgery as a result of rapidly developing spinal cord compression. The clinical conditions of the other eight patients (57\%) were more favourable, and conservative approaches were chosen. Ten patients showed clinical improvement by more than one point on AIS classification. 
Table 1: Demographic and clinical data of spontaneous spinal epidural haematoma group.

\begin{tabular}{|c|c|c|c|c|c|c|c|c|c|c|c|c|}
\hline $\mathrm{Pt}$ & Sex & Age & $\begin{array}{l}\text { Hyper } \\
\text { tension }\end{array}$ & $\begin{array}{l}\text { AC/AP } \\
\text { drugs }\end{array}$ & $\begin{array}{l}\text { Indication for } \\
\text { AC/AP drugs }\end{array}$ & $\begin{array}{l}\text { Clinical } \\
\text { signs } \\
\text { at onset }\end{array}$ & $\begin{array}{l}\text { Clinical neurological } \\
\text { status at onset }\end{array}$ & $\begin{array}{l}\text { Onset } \\
\text { (AIS) }\end{array}$ & $\begin{array}{l}\text { Level of } \\
\text { SSEH at } \\
\text { spinal } \\
\text { MRI }\end{array}$ & $\begin{array}{l}\text { Time to } \\
\text { operation }\end{array}$ & $\begin{array}{l}\text { Type of } \\
\text { treatment }\end{array}$ & $\begin{array}{l}\text { Clinical } \\
\text { outcome } \\
\text { (AIS) }\end{array}$ \\
\hline 1 & $M$ & 59 & Yes & $\begin{array}{l}\text { Warfarin } \\
\text { INR 3,2 }\end{array}$ & Atrial fibrillation & \begin{tabular}{|l} 
Sudden \\
pain in low \\
cervical \\
spine
\end{tabular} & $\begin{array}{l}\text { Hemiparesis with } \\
\text { progression to } \\
\text { quadriparesis and } \\
\text { hypaesthesia }\end{array}$ & C & $\begin{array}{l}\text { C5-T1 } \\
\text { ventral }\end{array}$ & 1 day & $\begin{array}{l}\text { Operation at } \\
\text { C5-T1 }\end{array}$ & $E$ \\
\hline 2 & $\mathrm{~F}$ & 68 & Yes & $\begin{array}{l}\text { Warfarin } \\
\text { INR } 2,4\end{array}$ & Atrial fibrillation & $\begin{array}{l}\text { Pain in } \\
\text { cervical } \\
\text { region }\end{array}$ & $\begin{array}{l}\text { Qvadriparesthesis, } \\
\text { transient } \\
\text { quadriparesis }\end{array}$ & $D$ & $\begin{array}{l}\text { C1-C6 } \\
\text { dorsal, } \\
\text { C1-C3 } \\
\text { ventral }\end{array}$ & None & Conservative & $E$ \\
\hline 3 & $\mathrm{~F}$ & 59 & Yes & $\begin{array}{l}\text { Warfarin } \\
\text { INR 3,4 }\end{array}$ & Atrial fibrillation & $\begin{array}{l}\text { Pain in low } \\
\text { cervical } \\
\text { spine }\end{array}$ & Qvadriparesthesis & $D$ & $\begin{array}{l}\text { C6-T1 } \\
\text { dorsal }\end{array}$ & None & Conservative & $E$ \\
\hline 4 & $\mathrm{~F}$ & 68 & Yes & No & No & $\begin{array}{l}\text { Pain in } \\
\text { cervical- } \\
\text { thoracic } \\
\text { region }\end{array}$ & $\begin{array}{l}\text { Slight right-sided } \\
\text { hemiparesis }\end{array}$ & $D$ & $\begin{array}{l}\text { C3-T5 } \\
\text { dorsal }\end{array}$ & None & Conservative & $E$ \\
\hline 5 & $\mathrm{~F}$ & 64 & Yes & $\begin{array}{l}\text { ASA } \\
100 \mathrm{mg} / \\
\text { day }\end{array}$ & Atherosclerosis & \begin{tabular}{|l} 
Sudden \\
pain in \\
thoracic \\
region after \\
Valsalva \\
manoeuvre \\
(sneezing) $^{2}$
\end{tabular} & $\begin{array}{l}\text { At onset } \\
\text { quadriplegia with } \\
\text { anaesthesia and } \\
\text { retention of urine }\end{array}$ & $A$ & $\begin{array}{l}\text { T6-T11 } \\
\text { dorsal }\end{array}$ & None & Conservative & $E$ \\
\hline 6 & $M$ & 89 & Yes & $\begin{array}{l}\text { Warfarin } \\
\text { INR } 2,8\end{array}$ & Atrial fibrillation & $\begin{array}{l}\text { Pain in the } \\
\text { lower back }\end{array}$ & $\begin{array}{l}\text { Slight paraparesis } \\
\text { with retention of } \\
\text { urine }\end{array}$ & $D$ & $\begin{array}{l}\text { L1 } \\
\text { ventral }\end{array}$ & None & Conservative & E \\
\hline 7 & $F$ & 84 & Yes & $\begin{array}{l}\text { Dabigatran } \\
220 \mathrm{mg} / \\
\text { day }\end{array}$ & Atrial fibrillation & $\begin{array}{l}\text { Pain in the } \\
\text { lower back }\end{array}$ & Normal & $E$ & $\begin{array}{l}\text { T12-L1 } \\
\text { ventral }\end{array}$ & None & Conservative & E \\
\hline 8 & $M$ & 89 & Yes & No & No & $\begin{array}{l}\text { Pain in the } \\
\text { lower back }\end{array}$ & Normal & $E$ & $\begin{array}{l}\text { L2 } \\
\text { dorsal }\end{array}$ & None & Conservative & E \\
\hline 9 & $\mathrm{~F}$ & 17 & No & No & No & $\begin{array}{l}\text { Pain in } \\
\text { cervical- } \\
\text { thoracic } \\
\text { region for a } \\
\text { week } \\
\end{array}$ & $\begin{array}{l}\text { Mild paraparesis } \\
\text { more pronounced } \\
\text { on the left side, } \\
\text { loss of sensation } \\
\text { from } \mathrm{T} 1\end{array}$ & C & $\begin{array}{l}\text { C7-T2 } \\
\text { dorsal }\end{array}$ & 5 days & $\begin{array}{l}\text { Operation at } \\
\text { C7-T2 }\end{array}$ & $\mathrm{D}$ \\
\hline 10 & $\mathrm{~F}$ & 67 & Yes & $\begin{array}{l}\text { Warfarin } \\
\text { INR 5,2 }\end{array}$ & $\begin{array}{l}\text { Deep vein } \\
\text { thrombosis }\end{array}$ & $\begin{array}{l}\text { Sudden } \\
\text { onset of } \\
\text { pain in } \\
\text { cervical- } \\
\text { thoracic } \\
\text { region }\end{array}$ & $\begin{array}{l}\text { Mild motor } \\
\text { paraparesis }\end{array}$ & C & $\begin{array}{l}\text { T2-T6 } \\
\text { dorsal }\end{array}$ & 2 days & $\begin{array}{l}\text { Operation at } \\
\text { C7-T6 }\end{array}$ & C \\
\hline 11 & $F$ & 68 & Yes & $\begin{array}{l}\text { Warfarin } \\
\text { INR 1,63 }\end{array}$ & $\begin{array}{l}\text { Deep vein } \\
\text { thrombosis }\end{array}$ & $\begin{array}{l}\text { Pain in the } \\
\text { left hip }\end{array}$ & $\begin{array}{l}\text { Severe motor } \\
\text { paraparesis }\end{array}$ & $B$ & $\begin{array}{l}\text { T11-L4 } \\
\text { dorsal }\end{array}$ & 4 days & $\begin{array}{l}\text { Operation at } \\
\text { T11-L4 }\end{array}$ & C \\
\hline 12 & $\mathrm{~F}$ & 61 & No & No & $\begin{array}{l}\text { Pulmonary } \\
\text { embolism, deep } \\
\text { vein thrombosis }\end{array}$ & $\begin{array}{l}\text { Pain in low } \\
\text { cervical } \\
\text { spine }\end{array}$ & $\begin{array}{l}\text { Dysesthesia, } \\
\text { hypaesthesia on } \\
\text { the right hand } \\
\text { with slight motor } \\
\text { weakness of C7 }\end{array}$ & D & $\begin{array}{l}\mathrm{C} 6-\mathrm{C} 7 \\
\text { ventral }\end{array}$ & None & Conservative & $\mathrm{D}$ \\
\hline 13 & $M$ & 42 & No & $\begin{array}{l}\text { Apixaban } \\
2 \times 5 \mathrm{mg} / \\
\text { day }\end{array}$ & $\begin{array}{l}\text { Deep vein } \\
\text { thrombosis }\end{array}$ & $\begin{array}{l}\text { Mild pain } \\
\text { in cervical } \\
\text { region }\end{array}$ & $\begin{array}{l}\text { Mild quadriparesis } \\
\text { with hypaesthesia } \\
\text { form C7 }\end{array}$ & C & $\begin{array}{l}\text { C4-C7 } \\
\text { dorsal }\end{array}$ & 1 day & $\begin{array}{l}\text { Operation at } \\
\text { C3-C6 }\end{array}$ & $\mathrm{D}$ \\
\hline 14 & $\mathrm{~F}$ & 77 & Yes & $\begin{array}{l}\text { ASA } \\
100 \mathrm{mg} / \\
\text { day }\end{array}$ & $\begin{array}{l}\text { Subrenal aortal } \\
\text { aneurysm }\end{array}$ & $\begin{array}{l}\text { Sudden } \\
\text { onset of } \\
\text { pain in } \\
\text { thoracic- } \\
\text { lumbar } \\
\text { region }\end{array}$ & $\begin{array}{l}\text { Paraplegia with } \\
\text { partial loss of } \\
\text { sensation from } \mathrm{T} 10\end{array}$ & A & $\begin{array}{l}\text { T9-T12 } \\
\text { dorsal }\end{array}$ & 1 day & $\begin{array}{l}\text { Operation at } \\
\text { T9-L1 }\end{array}$ & C \\
\hline
\end{tabular}


Of note, patient 5 developed paraparesis after sneezing, ${ }^{2}$ clinical improvement to normal status was observed after 24 hours, and patient 9 was pregnant.

AC: anticoagulation; AIS: American Spinal Injury Association (ASIA) Impairment Scale (AIS); AP: antiplatelet; ASA: acetylsalicylic acid; INR: international normalised ratio; pt: patient; SSEH: spontaneous spinal epidural haematoma.

\section{CONCLUSION}

SSEH is a rare entity concerning the expansion of blood collection in the spinal canal without clear traumatic or iatrogenic causes, with the prognosis affected by the severity of spinal cord compression, delayed final diagnosis, proper timing in surgical intervention, and wellchosen conservative approach in spontaneously improving cases. ${ }^{3,4}$ In this study, one of the most important findings was that $71 \%$ of patients (mostly in those who were of an older age) received oral anticoagulation/antiplatelet treatment and $57 \%$ of patients with slight or mild neurological symptoms were successfully treated with a conservative approach.

\section{References}

1. Kirazli $Y$ et al Spinal epidural hematoma associated with oral anticoagulation therapy. Am J Phys Med Rehabil. 2004;83:220-3.

2. Stetkarova I et al. Spontaneous spinal epidural hematoma after abrupt sneezing with prompt recovery of severe paraparesis. Am J Emerg Med. 2014;32(12):1555.e3-5.

3. Sirin $\mathrm{S}$ et al. Is spontaneous spinal epidural hematoma in elderly patients an emergency surgical case? Turk Neurosurg. 2010;20:557-60

4. Eto $\mathrm{F}$ et al. Clinical features of spontaneous spinal epidural hematoma. J Rural Med. 2019;14:206-10. 